\title{
An enhanced spatial smoothing technique with ESPRIT algorithm for direction of arrival estimation in coherent scenarios
}

\author{
Jingjing Pan, Meng Sun, Yide Wang, Xiaofei Zhang
}

\begin{abstract}
Subspace-based methods suffer from the rank loss of the noise free data covariance matrix in the context of direction of arrival (DOA) estimation of coherent sources. The well-known spatial smoothing techniques are then widely employed to create a rank restored data covariance matrix. However, conventional spatial smoothing techniques, such as the spatial smoothing pre-processing (SSP), modified spatial smoothing pre-processing (MSSP), and improved spatial smoothing (ISS), do not make full use of the available information in the data covariance matrix. In this paper, an enhanced spatial smoothing (ESS) technique is proposed to exploit both the covariance matrices of individual subarrays and the cross-covariance matrices of different subarrays. Besides, the proposed method can work directly on the signal subspace (ESS-SS), since the signal subspace contains all the information of the DOAs of incoming signals. After decorrelation, the subspace method ESPRIT is adopted to estimate the DOAs. Compared with conventional approaches, the proposed method is more powerful to de-correlate the correlation between signals, and also more robust to the noise impact. The proposed method is tested on numerical data in coherent scenarios, and compared with conventional approaches. Simulation results show that the proposed method has an enhanced resolving capability and a lower signal-to-noise ratio threshold.
\end{abstract}

Index Terms - Direction of arrival (DOA) estimation, enhanced spatial smoothing (ESS), signal subspace.

\section{INTRODUCTION}

Direction of arrival (DOA) estimation is a major issue in array signal processing [1], [2] and practical engineering applications, e.g., radar, sonar, navigation, non-destructive testing, and wireless communications [3], [4], [5]. A variety of array signal processing methods have been proposed for DOA estimation. Since the 1970s, a class of eigenstructure-based methods, called subspace-based methods or high-resolution methods, have been developed and become very popular, such as multiple signal classification (MUSIC) [6], estimation of signal parameters via rotational invariance techniques (ESPRIT) [7], matrix pencil method (MPM) [8], and subspace fitting method [9], [10]. These methods have been proven to have higher resolution capability than conventional Fourierbased methods, the Capon method [11], and linear-predictionbased methods [12]. Among the subspace-based methods, MUSIC and ESPRIT are the two leading methods. In the 1990s, propagator-based methods or linear subspace methods, such as orthogonal propagator method (OPM) [13], were proposed for the estimation of DOA, which use linear operations

Manuscript received 2019. instead of eigenvalue decomposition (EVD) or singular value decomposition (SVD).

Both subspace-based and propagator-based methods were originally proposed for uncorrelated signals, they are based on the assumption of the non-singularity of the source covariance matrix. In reality, there are practical applications where coherent signals are presented because of multi-path propagation by different reflective media or deliberate jamming in military scenarios [14], [15]. In this case, the cross-correlation between signals may be high enough to degrade the performance of the aforementioned methods, since there is a rank loss in the source covariance matrix. The authors of [16] proposed a generalized MUSIC algorithm for DOA estimation of coherent signals, and subspace fitting methods [9], [10] can also handle the case of coherent signals in DOA estimation. Unfortunately, both the generalized MUSIC and subspace fitting methods require multi-dimensional search, which is computationally expensive for real-time applications.

To counter the deleterious effect of the coherency between signals, a number of techniques have been developed to decorrelate the correlation between signals. The well-known spatial smoothing pre-processing (SSP) technique was proposed in [17] and modified in [14], [18], [19] (modified SSP or MSSP), which partitions the entire observation array into a series of overlapping subarrays to obtain a new data covariance matrix with restored rank. The subspace-based method MPM can directly solve the problem of coherent signals [8]. Its principle is to first construct a Hankel matrix of the received signals, which is equivalent to the spatial smoothing approach, and then use the generalized idea of the pencil function for DOA estimation. The spatial-smoothing-based techniques have achieved numerous results, and many improvements based on SSP have been proposed [20]-[21].

In addition, there are many matrix-reconstruction-based approaches for coherent signals. The authors of [22], [23] proposed the construction of new matrices, whose ranks are independent of the coherency between signals in DOA estimation. In [22], the authors attempted to reconstruct a Toeplitz matrix from the data covariance matrix in order to estimate DOA. This method is not affected by the coherency between signals and shows reasonable performance, but with a reduced array aperture. To achieve a larger effective array aperture, a non-Toeplitz matrix was used in [23] to resolve more signals than the number of arrays under the coexistence of coherent and incoherent signals. In [24], the authors proposed to modify the received signal model with a multi-invariance property and 
apply multi-invariance ESPRIT or MUSIC for coherent DOA estimation.

Within the aforementioned de-correlation methods, two improved spatial smoothing (ISS) techniques [25], [26] have drawn much attention [27], [28], [29], [30]. The two ISS techniques exploit not only the covariance matrices of individual subarrays, but also the cross-covariance matrices of different subarrays. In contrast, the conventional SSP only uses the covariance matrices of individual subarrays [25]. The ISS techniques have been proved to be more powerful to decorrelate the correlation between signals than the conventional SSP [25], [26].

However, the two ISS techniques [25], [26] do not use all the information from the received signals (details are given in Section 3). In this paper, an enhanced spatial smoothing technique (called ESS) is proposed, which takes the full advantage of the covariance matrices of individual subarrays as well as the cross-covariance matrices of different subarrays. Furthermore, the proposed method can be operated on the signal subspace instead of the entire data covariance matrix, since the signal subspace contains all the information of the signals [1]. Then, the subspace-based method, ESPRIT, is applied for DOA estimation in coherent scenarios, after the proposed de-correlation pre-processing. Compared with conventional approaches, the proposed method has two major merits: 1) it fully utilizes the information coming from the signal subspace, which is more powerful for de-correlation, 2 ) it is more robust to noise. Performance analysis of the proposed method on DOA estimation of coherent sources is provided with numerical data, which proves the effectiveness of the proposed method.

The rest of this paper is organized as follows. Far field narrow band signal model is presented in Section 2. In Section 3, the principle of the conventional SSP and two ISS techniques [25], [26] are briefly reviewed. In Section 4, the proposed ESS technique is described. Simulation results and a discussion on the performance of the ESPRIT algorithm with the two ISS techniques presented in [25], [26], and the proposed method are provided in Section 5. Conclusions and perspectives are given in Section 6 .

\section{Signal MODEL}

Consider $K$ far field narrow band signals impinging on a uniform linear array (ULA) with $N$ isotropic antenna elements. The received signals are coherent. The index of the antennas is set to be $0,1, \ldots, N-1$, as shown in Fig. 1, the 0th sensor is set to be the reference sensor. The received signal for the $m$ th sensor $(m=0,1, \ldots, N-1)$ at time $t$ ( $t=1,2, \ldots, T_{k}$, with $T_{k}$ the number of snapshots, $t$ is normalized with the sampling period) can be written as

$$
r_{m}(t)=\sum_{k=1}^{K} s_{k}(t) \exp \left(-j 2 m \pi \frac{d}{\lambda} \sin \theta_{k}\right)+n_{m}(t)
$$

where $s_{k}(t)$ is the complex envelope of the $k$ th signal with corresponding DOA $\theta_{k}$ received at the 0th sensor, $n_{m}(t)$ is the additive white Gaussian noise (AWGN) at the corresponding

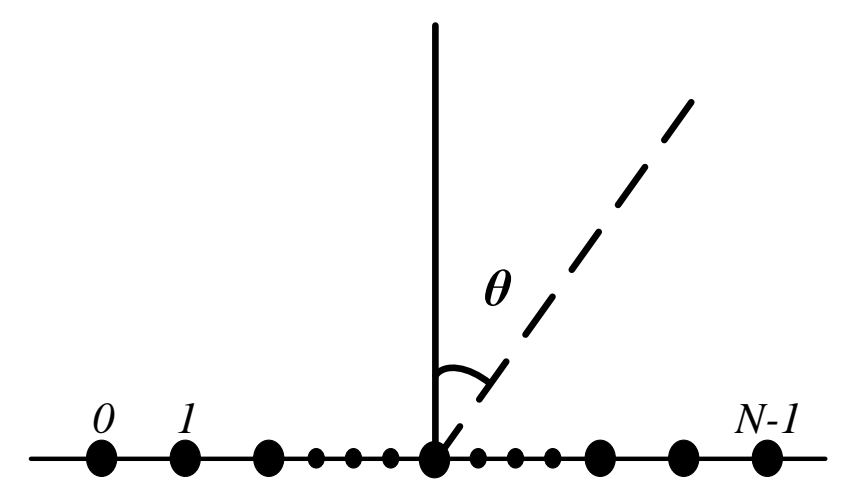

Fig. 1. Uniform Linear Array.

sensor with zero mean and variance $\sigma^{2}, d$ denotes the distance between two adjacent sensors, and $\lambda$ is the wavelength of incoming signals.

(1) can be written in the following vector form:

$$
\mathbf{r}(t)=\mathbf{A s}(t)+\mathbf{n}(t)
$$

with the following notation definitions:

- $\mathbf{r}(t)=\left[r_{0}(t) r_{1}(t) \cdots r_{N-1}(t)\right]^{T}$ is the $(N \times 1)$ received signal vector; the superscript $T$ denotes the transpose operation;

- $\mathbf{A}=\left[\begin{array}{llll}\mathbf{a}\left(\theta_{1}\right) & \mathbf{a}\left(\theta_{2}\right) & \ldots & \mathbf{a}\left(\theta_{K}\right)\end{array}\right]$ is the $(N \times K)$ directional matrix;

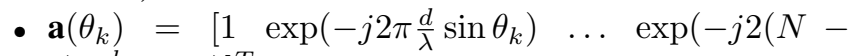
1) $\left.\left.\pi \frac{d}{\lambda} \sin \theta_{k}\right)\right]^{T}$ is the directional vector;

- $\mathbf{s}(t)=\left[\begin{array}{llll}s_{1}(t) & s_{2}(t) & \cdots & s_{K}(t)\end{array}\right]^{T}$ is the $(K \times 1)$ source vector;

- $\mathbf{n}(t)=\left[n_{0}(t) n_{1}(t) \cdots n_{N-1}(t)\right]^{T}$ is the $(N \times 1)$ noise vector, with zero mean and covariance matrix $\sigma^{2} \mathbf{I}$; $\mathbf{I}$ is the $(N \times N)$ identity matrix.

According to signal model (2) and assuming that the noise is independent from the signals, the data covariance matrix $\mathbf{R}$ can be written as

$$
\begin{aligned}
\mathbf{R} & =E\left\{\mathbf{r}(t) \mathbf{r}^{H}(t)\right\} \\
& =\mathbf{A} E\left\{\mathbf{s}(t) \mathbf{s}^{H}(t)\right\} \mathbf{A}^{H}+E\left\{\mathbf{n}(t) \mathbf{n}^{H}(t)\right\} \\
& =\mathbf{A} \mathbf{S} \mathbf{A}^{H}+\sigma^{2} \mathbf{I},
\end{aligned}
$$

where $E\{:\}$ denotes the mathematical expectation, $\mathbf{S}$ is the $(K \times K)$-dimensional covariance matrix of the source vector $\mathbf{s}(t)$, and the superscript $H$ denotes the conjugate transpose operation. Matrix $\mathbf{S}$ is full rank when the received signals are not fully correlated. However, when the received signals are fully correlated (coherent), matrix $\mathbf{S}$ is singular (rank loss phenomena) [14], [16].

By applying EVD, the data covariance matrix $\mathbf{R}$ can be reformulated in terms of its eigenvalues and associated eigenvectors:

$$
\mathbf{R}=\mathbf{U} \boldsymbol{\Lambda} \mathbf{U}^{H}
$$

where $\boldsymbol{\Lambda}=\operatorname{diag}\left\{\lambda_{1}, \lambda_{2}, \ldots, \lambda_{N}\right\}$ is a diagonal matrix of eigenvalues $\left(\lambda_{1} \geq \lambda_{2} \geq \lambda_{3} \geq \cdots \geq \lambda_{N}\right)$, and their associated eigenvectors are in the columns of $\mathbf{U}=\left\{\mathbf{u}_{1}, \mathbf{u}_{2}, \ldots, \mathbf{u}_{N}\right\}$. Consequently, the data covariance matrix $\mathbf{R}$ can be decom- 
posed into

$$
\begin{aligned}
\mathbf{R} & =\boldsymbol{\Gamma}_{s}+\boldsymbol{\Gamma}_{n} \\
& =\mathbf{U}_{s} \boldsymbol{\Lambda}_{s} \mathbf{U}_{s}^{H}+\mathbf{U}_{n} \boldsymbol{\Lambda}_{n} \mathbf{U}_{n}^{H},
\end{aligned}
$$

where $\boldsymbol{\Lambda}_{s}$ is a diagonal matrix containing the $K$ maximum eigenvalues $\lambda_{1} \geq \cdots \geq \lambda_{K}>\sigma^{2}$; and their associated eigenvectors are in the columns of $\mathbf{U}_{s}$, which span the signal subspace; $\boldsymbol{\Lambda}_{n}$ is also a diagonal matrix that contains the $N-K$ small eigenvalues $\lambda_{K+1}=\cdots=\lambda_{N}=\sigma^{2}$; and their associated eigenvectors are in the columns of $\mathbf{U}_{n}$, which span the noise subspace.

Nevertheless, in coherent scenarios, only 1 eigenvalue is larger than the noise variance, i.e., $\lambda_{1}>\lambda_{2}=\lambda_{3}=\cdots=$ $\lambda_{N}=\sigma^{2}$. Therefore, $\boldsymbol{\Lambda}_{s}$ is a scalar $\left(\lambda_{1}\right)$ and $\mathbf{U}_{s}$ is a vector $\left(\mathbf{u}_{1}\right)$. Matrix $\boldsymbol{\Gamma}_{s}$ can be rewritten as

$$
\boldsymbol{\Gamma}_{s}=\mathbf{U}_{s} \boldsymbol{\Lambda}_{s} \mathbf{U}_{s}^{H}=\lambda_{1} \mathbf{u}_{1} \mathbf{u}_{1}^{H} .
$$

Therefore, the rank of $\boldsymbol{\Gamma}_{s}$ drops to 1 . In this case, methods such as MUSIC and ESPRIT cannot be directly applied, because they are based on the full rank property of matrix $\boldsymbol{\Gamma}_{s}$.

\section{SPATIAL SMOOTHING TECHNIQUE}

As mentioned previously, the main obstacle to apply subspace-based and propagator-based methods is the coherency between the received signals. In this section, the de-correlation process of the conventional spatial smoothing technique [17], [18] and two ISS techniques [25], [26] are briefly reviewed.

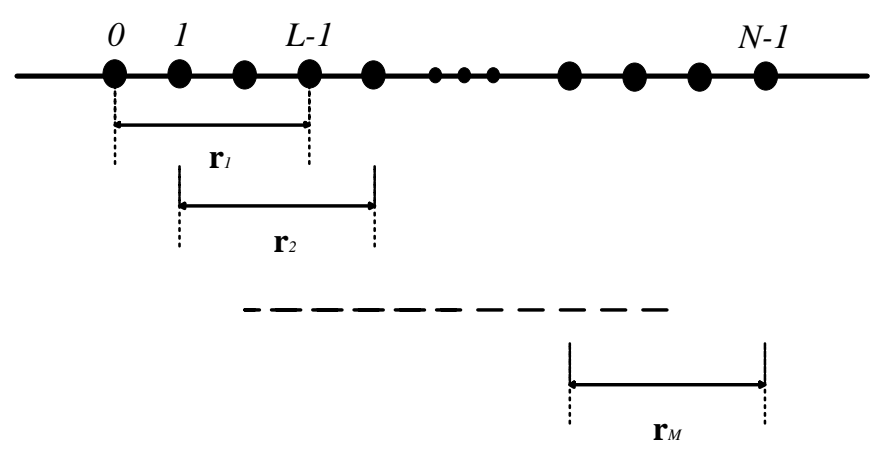

Fig. 2. Overlapping subarrays for spatial smoothing techniques.

The conventional way of mitigating the influence of crosscorrelation magnitude is to apply spatial-smoothing-based techniques on the data covariance matrix $\mathbf{R}$. As shown in Fig. 2, the entire array is partitioned into $M$ overlapping subarrays, each consisting of $L$ elements. Therefore, it is easy to verify that the maximum number of signals that can be estimated is $L-1$. Parameters $N, L$, and $M$ are related to each others by

$$
N=L+M-1 \text {. }
$$

Let $\mathbf{r}_{i}$ denote the $(L \times 1)$ received signal vector on the $i$ th subarray, it can be written as

$$
\mathbf{r}_{i}=\mathbf{A}_{1} \mathbf{D}^{i-1} \mathbf{s}+\mathbf{n}_{i}
$$

where $\mathbf{n}_{i}$ is the $(L \times 1)$ noise vector on the $i$ th subarray; the $(L \times K)$ matrix $\mathbf{A}_{1}$ denotes the first $L$ rows of the directional matrix $\mathbf{A}$, which is independent of parameter $i$; $\mathbf{D}$ is a $(K \times K)$ diagonal matrix:

$\mathbf{D}=\operatorname{diag}\left\{\exp \left\{-j 2 \pi \frac{d}{\lambda} \sin \theta_{1}\right\}, \ldots, \exp \left\{-j 2 \pi \frac{d}{\lambda} \sin \theta_{K}\right\}\right\}$.

Therefore, the noiseless cross-covariance matrix of the $i$ th and $j$ th subarrays $\mathbf{R}_{i j}$ can be written as

$$
\begin{aligned}
\mathbf{R}_{i j} & =E\left\{\mathbf{r}_{i}(t) \mathbf{r}_{j}^{H}(t)\right\} \\
& =\mathbf{A}_{1} \mathbf{D}^{i-1} \mathbf{S}\left(\mathbf{D}^{j-1}\right)^{H} \mathbf{A}_{1}^{H} .
\end{aligned}
$$

Similarly, after some mathematical manipulations, the backward cross-covariance matrix between the $i$ th and $j$ th subarrays can be defined as

$$
\begin{aligned}
\overline{\mathbf{R}}_{i j} & =\mathbf{J} E\left\{\mathbf{r}_{i}^{*}(t) \mathbf{r}_{j}^{T}(t)\right\} \mathbf{J} \\
& =\mathbf{J} \mathbf{R}_{i j}^{*} \mathbf{J},
\end{aligned}
$$

where $\mathbf{J}$ is the $(L \times L)$ anti-identity matrix (exchange matrix) and the operator $*$ denotes the complex conjugate.

According to [14], the rank restored data covariance matrix $\mathbf{R}_{\text {SSP }}$ after SSP is

$$
\mathbf{R}_{\mathrm{SSP}}=\frac{1}{M} \sum_{i=1}^{M} \mathbf{R}_{i i}
$$

MSSP is an extension of SSP, which utilizes the covariance matrix of the $i$ th forward and backward subarrays to construct the rank restored data covariance matrix $\mathbf{R}_{\mathrm{MSSP}}$ as follows [18]:

$$
\mathbf{R}_{\mathrm{MSSP}}=\frac{1}{2 M} \sum_{i=1}^{M}\left\{\mathbf{R}_{i i}+\overline{\mathbf{R}}_{i i}\right\}
$$

Unlike the conventional SSP and MSSP, the authors of [25], [26] proposed two different ISS techniques, which are based on quadratic spatial smoothing and yield better angular resolution for coherent signals. The method proposed in [25], called ISS1 in this paper, takes advantage of all the cross-covariance matrices $\mathbf{R}_{i j}$ and part of the covariance matrices $\mathbf{R}_{i i} / \mathbf{R}_{j j}$. ISS 1 constructs the following rank restored data covariance matrix $\mathbf{R}_{\mathrm{ISS} 1}$ :

$$
\mathbf{R}_{\mathrm{ISS} 1}=\frac{1}{2 M} \sum_{i=1}^{M} \sum_{j=1}^{M}\left\{\mathbf{R}_{i j} \mathbf{R}_{j i}+\overline{\mathbf{R}}_{i j} \overline{\mathbf{R}}_{j i}\right\} .
$$

The method proposed in [26], called ISS2 in this paper, takes advantage of all the covariance matrices $\mathbf{R}_{i i} / \mathbf{R}_{j j}$, by using the following rank restored matrix:

$$
\mathbf{R}_{\mathrm{ISS} 2}=\frac{1}{2 M} \sum_{i=1}^{M} \sum_{j=1}^{M}\left\{\mathbf{R}_{i i} \mathbf{R}_{j j}+\overline{\mathbf{R}}_{i i} \overline{\mathbf{R}}_{j j}\right\}
$$

It can be seen from (12) and (13) that neither ISS1 nor ISS2 uses all the information from the subarrays. In (12), ISS1 does not use the information from $\mathbf{R}_{i i} \mathbf{R}_{j j}$ for $i \neq j$; all the crosscovariance matrices $\mathbf{R}_{i j}, i \neq j$ are missing in ISS2 (13).

\section{ENHANCED SPATIAL SMOOTHING TECHNIQUE}

As shown in the previous section, one drawback of the two ISS techniques is that they do not fully utilize the 
information from the subarrays. Therefore, in this section, the ESS technique is proposed. The proposed rank restored data covariance matrix $\mathbf{R}_{\mathrm{ESS}}$ after ESS including all the crosscovariance matrices of different subarrays $\mathbf{R}_{i j}$ and covariance matrices of individual subarrays $\mathbf{R}_{i i} / \mathbf{R}_{j j}$, is expressed as

$$
\begin{aligned}
\mathbf{R}_{\mathrm{ESS}}= & \frac{1}{2 M} \sum_{i=1}^{M} \sum_{j=1}^{M}\left\{\left(\mathbf{R}_{i j} \mathbf{R}_{j i}+\overline{\mathbf{R}}_{i j} \overline{\mathbf{R}}_{j i}\right)\right. \\
& \left.+\left(\mathbf{R}_{i i} \mathbf{R}_{j j}+\overline{\mathbf{R}}_{i i} \overline{\mathbf{R}}_{j j}\right)\right\} .
\end{aligned}
$$

As shown in Appendix $A$, the rank of the source covariance matrix after the proposed ESS technique is equal to $K$. Therefore, it is clear that the proposed method can restore the rank of the data covariance matrix.

As mentioned in Section 2, only the signal subspace contains the information of the received signals. Nevertheless, the conventional spatial-smoothing-based techniques are applied on the data covariance matrix. The proposed ESS technique can also work on the signal subspace, called ESS-SS in this paper. As discussed before, for coherent signals, $\boldsymbol{\Lambda}_{s}=\lambda_{1}$ is a scalar, and $\mathbf{U}_{s}=\mathbf{u}_{1}$ is an $(N \times 1)$ vector. The received data covariance matrix $\mathbf{R}$ can then be written as

$$
\mathbf{R}=\lambda_{1} \mathbf{u}_{1} \mathbf{u}_{1}^{H}+\sigma^{2} \mathbf{U}_{n} \mathbf{U}_{n}^{H} .
$$

Since

$$
\mathbf{u}_{1} \mathbf{u}_{1}^{H}+\mathbf{U}_{n} \mathbf{U}_{n}^{H}=\mathbf{I},
$$

we have

$$
\begin{aligned}
\mathbf{A S A}^{H} & =\mathbf{R}-\sigma^{2} \mathbf{I} \\
& =\lambda_{1} \mathbf{u}_{1} \mathbf{u}_{1}^{H}+\sigma^{2} \mathbf{U}_{n} \mathbf{U}_{n}^{H}-\sigma^{2} \mathbf{I} \\
& =\left(\lambda_{1}-\sigma^{2}\right) \mathbf{u}_{1} \mathbf{u}_{1}^{H},
\end{aligned}
$$

therefore, we can deduce that

$$
\begin{aligned}
\mathbf{u}_{1} & =\frac{1}{\lambda_{1}-\sigma^{2}} \mathbf{A S A}^{H} \mathbf{u}_{1} \\
& =\mathbf{A t}
\end{aligned}
$$

where $\mathbf{t}=\frac{1}{\lambda_{1}-\sigma^{2}} \mathbf{S} \mathbf{A}^{H} \mathbf{u}_{1}$ is a $(K \times 1)$ vector. The expression of matrix $\boldsymbol{\Gamma}_{s}$ is different for coherent signals and for nofully correlated signals. For coherent signals, $\boldsymbol{\Gamma}_{s}=\lambda_{1} \mathbf{u}_{1} \mathbf{u}_{1}^{H}$; for no-fully correlated signals, $\boldsymbol{\Gamma}_{s}=\mathbf{U}_{s} \boldsymbol{\Lambda}_{s} \mathbf{U}_{s}^{H}$. In the following, matrix $\boldsymbol{\Gamma}_{s}$ is normalized, index 1 in $\mathbf{u}_{1}$ and index $s$ in $\boldsymbol{\Gamma}_{s}$ are dropped for notational convenience.

We define the $i$ th and $j$ th subarrays of matrix $\Gamma$ for coherent signals as

$$
\boldsymbol{\Gamma}_{i j}=\mathbf{v}_{i} \mathbf{v}_{j}^{H}
$$

where $\mathbf{v}_{i}=\mathbf{A}_{1} \mathbf{D}^{i-1} \mathbf{t}$ is the $(L \times 1)$ vector. For no-fully correlated signals, according to [1], $\mathbf{U}_{s}=\mathbf{A T}, \mathbf{T}$ is $(K \times K)$ dimensional invertible matrix. The $i$ th and $j$ th subarrays of matrix $\boldsymbol{\Gamma}$ for no-fully correlated signals can be expressed as

$$
\begin{aligned}
\boldsymbol{\Gamma}_{i j} & =\mathbf{A}_{1} \mathbf{D}^{i-1} \mathbf{T} \boldsymbol{\Lambda}_{s} \mathbf{T}^{H}\left(\mathbf{D}^{j-1}\right)^{H} \mathbf{A}_{1}^{H} \\
& =\mathbf{v}_{i} \boldsymbol{\Lambda}_{s} \mathbf{v}_{j}^{H},
\end{aligned}
$$

where $\mathbf{v}_{i}$ in (19) can be written as $\mathbf{v}_{i}=\mathbf{A}_{1} \mathbf{D}^{i-1} \mathbf{T}$. Then, we apply the proposed ESS technique on the signal subspace. In the following, only the coherent signals are studied, the case of no-fully correlated signals is just a straightforward extension. The rank restored covariance matrix $\mathbf{R}_{\mathrm{ESS}-\mathrm{SS}}$ can be reformulated as follows:

$$
\begin{aligned}
\mathbf{R}_{\text {ESS-SS }}= & \frac{1}{2 M} \sum_{i=1}^{M} \sum_{j=1}^{M}\left\{\left(\boldsymbol{\Gamma}_{i j} \boldsymbol{\Gamma}_{j i}+\overline{\boldsymbol{\Gamma}}_{i j} \overline{\boldsymbol{\Gamma}}_{j i}\right)\right. \\
& \left.+\left(\boldsymbol{\Gamma}_{i i} \boldsymbol{\Gamma}_{j j}+\overline{\boldsymbol{\Gamma}}_{i i} \overline{\boldsymbol{\Gamma}}_{j j}\right)\right\} .
\end{aligned}
$$

To facilitate the expression of the proposed ESS-SS technique, the forward only smoothed covariance matrix is taken as an example, which can be expressed as

$$
\mathbf{R}_{\mathrm{ESS}-\mathrm{SS}}^{f}=\frac{1}{2 M} \sum_{i=1}^{M} \sum_{j=1}^{M}\left\{\boldsymbol{\Gamma}_{i j} \boldsymbol{\Gamma}_{j i}+\boldsymbol{\Gamma}_{i i} \boldsymbol{\Gamma}_{j j}\right\} .
$$

More specifically, we define

$$
\begin{aligned}
& \mathbf{R}_{1}^{f}=\sum_{i=1}^{M} \sum_{j=1}^{M}\left\{\boldsymbol{\Gamma}_{i j} \boldsymbol{\Gamma}_{j i}\right\}=\sum_{i=1}^{M} \sum_{j=1}^{M}\left\{\mathbf{v}_{i} \mathbf{v}_{j}^{H} \mathbf{v}_{j} \mathbf{v}_{i}^{H}\right\} \\
& =\sum_{i=1}^{M} \sum_{j=1}^{M}\left\{\mathbf{A}_{1} \mathbf{D}^{i-1} \mathbf{t t}^{H}\left(\mathbf{D}^{j-1}\right)^{H} \mathbf{A}_{1}^{H} \mathbf{A}_{1} \mathbf{D}^{j-1} \mathbf{t t}^{H}\left(\mathbf{D}^{i-1}\right)^{H} \mathbf{A}_{1}^{H}\right\} \\
& =\mathbf{A}_{1}\left\{\sum_{i=1}^{M} \sum_{j=1}^{M} \mathbf{D}^{i-1} \mathbf{t} \mathbf{t}^{H}\left(\mathbf{D}^{j-1}\right)^{H} \mathbf{A}_{1}^{H} \mathbf{A}_{1} \mathbf{D}^{j-1} \mathbf{t t}^{H}\left(\mathbf{D}^{i-1}\right)^{H}\right\} \mathbf{A}_{1}^{H}
\end{aligned}
$$

and

$$
\begin{aligned}
& \mathbf{R}_{2}^{f}=\sum_{i=1}^{M} \sum_{j=1}^{M}\left\{\boldsymbol{\Gamma}_{i i} \boldsymbol{\Gamma}_{j j}\right\}=\sum_{i=1}^{M} \sum_{j=1}^{M}\left\{\mathbf{v}_{i} \mathbf{v}_{i}^{H} \mathbf{v}_{j} \mathbf{v}_{j}^{H}\right\} \\
& =\sum_{i=1}^{M} \sum_{j=1}^{M}\left\{\mathbf{A}_{1} \mathbf{D}^{i-1} \mathbf{t t}^{H}\left(\mathbf{D}^{i-1}\right)^{H} \mathbf{A}_{1}^{H} \mathbf{A}_{1} \mathbf{D}^{j-1} \mathbf{t t}^{H}\left(\mathbf{D}^{j-1}\right)^{H} \mathbf{A}_{1}^{H}\right\} \\
& =\mathbf{A}_{1}\left\{\sum_{i=1}^{M} \sum_{j=1}^{M} \mathbf{D}^{i-1} \mathbf{t t}^{H}\left(\mathbf{D}^{i-1}\right)^{H} \mathbf{A}_{1}^{H} \mathbf{A}_{1} \mathbf{D}^{j-1} \mathbf{t t}^{H}\left(\mathbf{D}^{j-1}\right)^{H}\right\} \mathbf{A}_{1}^{H}
\end{aligned}
$$

Similarly, the forward smoothed covariance matrix $\mathbf{R}_{\mathrm{ESS}-\mathrm{SS}}^{f}=\frac{1}{2 M}\left\{\mathbf{R}_{1}^{f}+\mathbf{R}_{2}^{f}\right\}$ also has a rank of $K$ as in the conventional methods. The proof is in Appendix $B$. Moreover, the proof for the forward-backward smoothed covariance matrix is just a straightforward extension of the above.

Compared with the conventional approaches, the proposed method takes full advantage of the signal subspace and is more robust to noise. With the rank restored data covariance matrix, the subspace-based method ESPRIT can then be applied for DOA estimation.

The ESPRIT algorithm divides the directional matrix $\mathbf{A}_{1}$ into two overlapping submatrices $\mathbf{A} \uparrow$ and $\mathbf{A} \downarrow$ with $((L-$ $1) \times K$ ) dimensions. Define $\mathbf{A} \uparrow$ and $\mathbf{A} \downarrow$ by deleting the last and first row from $\mathbf{A}_{1}$, respectively,

$$
\mathbf{A}_{1}=\left(\begin{array}{c}
\mathbf{A} \uparrow \\
\text { last row }
\end{array}\right)=\left(\begin{array}{c}
\text { first row } \\
\mathbf{A} \downarrow
\end{array}\right) .
$$

Therefore, $\mathbf{A} \uparrow$ and $\mathbf{A} \downarrow$ are related to each other by the $(K \times K)$ diagonal matrix $\mathbf{D}$, the elements of which depend on the DOA to be estimated as

$$
\mathbf{A} \downarrow=\mathbf{A} \uparrow \mathbf{D} .
$$

In theory, matrix $\mathbf{D}$ cannot be estimated from data. Ac- 


\begin{tabular}{|c|c|c|}
\hline Method & $\widehat{\theta_{1}}$ & $\widehat{\theta}_{2}$ \\
\hline ISS1 & $-6.02^{\circ}$ & $6.96^{\circ}$ \\
\hline ISS2 & $-5.85^{\circ}$ & $7.07^{\circ}$ \\
\hline ESS & $-5.93^{\circ}$ & $6.97^{\circ}$ \\
\hline ESS-SS & $-6.02^{\circ}$ & $6.99^{\circ}$ \\
\hline
\end{tabular}

CASE $a$, ESTIMATED DOAS OF SIGNALS BY ISS 1, ISS2, PROPOSED ESS, AND ESS-SS WITH A SINGLE RUN OF ESPRIT ALGORITHM; THE TRUE DOAS ARE $\theta_{1}=-6^{\circ}$ AND $\theta_{2}=7^{\circ}$.

cording to [7], on the basis of the EVD of the data covariance matrix, it can be shown that the diagonal elements of $\mathbf{D}$ can be retrieved from a similar matrix $\mathbf{X}$ (the detail is in Appendix $C$ ) having the same eigenvalues as $\mathbf{D}$. As such, the DOAs can be estimated by calculating the phase of the eigenvalues of this new matrix $\mathbf{X}$ :

$$
\hat{\theta}_{k}=-\arcsin \left\{\frac{\lambda}{2 \pi d} \angle x_{k}\right\}
$$

where $x_{k}$ is the $k$ th eigenvalue of $\mathbf{X}, \angle$ is the angle.

The general steps of the proposed ESS technique with the ESPRIT algorithm are summarized as follows:

- Estimate the data covariance matrix $\mathbf{R}$ from (3).

- Apply the EVD on R.

- Apply the proposed ESS technique on the signal subspace (ESS-SS).

- Estimate the DOAs by the ESPRIT algorithm.

\section{Simulations AND Discussion}

In this section, the performance of the proposed ESS technique combined with the ESPRIT algorithm is tested on simulated data with 5 different simulations. A ULA with $N=11$ isotropic sensors is considered, along with two farfield coherent narrow band signals $(K=2)$ with equal power. The number of subarrays is equal to $M=6$. Moreover, the distance between two adjacent sensors is equal to half of the wavelength with $\frac{d}{\lambda}=0.5$. In the simulations, the propagator method presented in [28] is applied to reduce the noise impact. Two cases are taken into account with different DOAs of incoming signals:

- Case $a . \theta_{1}=-6^{\circ}$ and $\theta_{2}=7^{\circ}$;

- Case b. $\theta_{1}=-2^{\circ}$ and $\theta_{2}=3^{\circ}$.

In the first simulation, the proposed ESS and ESS-SS are tested in a single run of ESPRIT algorithm. The estimation results of ISS1 [25] and ISS2 [26] are taken as comparisons. The signal-to-noise ratio (SNR) is fixed at $0 \mathrm{~dB}$. The data covariance matrix is estimated from 500 independent snapshots.

Tables I and II show the estimated DOAs of signals obtained by these methods for Cases $a$ and $b$. In a single run, the proposed ESS-SS can accurately detect the true DOAs of the incoming signals in both cases, compared with the proposed ESS, ISS1, and ISS2. However, the performance of the proposed method cannot be evaluated in a single run of the algorithm. To better show the merits of the proposed method, the statistical results of the proposed method are presented in the following.

\begin{tabular}{|c|c|c|}
\hline Method & $\widehat{\theta}_{1}$ & $\widehat{\theta}_{2}$ \\
\hline ISS1 & $-2.13^{\circ}$ & $2.94^{\circ}$ \\
\hline ISS2 & $-1.95^{\circ}$ & $3.25^{\circ}$ \\
\hline ESS & $-2.01^{\circ}$ & $3.12^{\circ}$ \\
\hline ESS-SS & $-2.01^{\circ}$ & $3.01^{\circ}$ \\
\hline
\end{tabular}

CASE $b$, estimated DOAS OF SIGNALS B ISS1, ISS2, PROPOSED ESS, AND ESS-SS WITH A SINGLE RUN OF ESPRIT ALGORITHM; THE TRUE DOAS ARE $\theta_{1}=-2^{\circ}$ AND $\theta_{2}=3^{\circ}$.

In the second simulation, the performance of the proposed method versus SNR is assessed with a Monte-Carlo process of 200 independent runs. The root-mean-square error (RMSE) of the estimated DOA is defined as follows:

$$
\mathrm{RMSE}=\sqrt{\frac{1}{K J} \sum_{k=1}^{K} \sum_{j=1}^{J}\left(\hat{\theta}_{k j}-\theta_{k}\right)^{2}},
$$

where $\hat{\theta}_{k j}$ denotes the estimated DOA of the $k$ th incoming signal for the $j$ th run of the algorithm, and $J$ is the total number of Monte-Carlo trials. SNR varies from -15 to 10 $\mathrm{dB}$. The statistical performance of all the methods in both cases is presented. Moreover, the Cramér-Rao Bound (CRB) results are also provided [31].

Figs. 3 and 4 plot the RMSEs of DOA estimation against

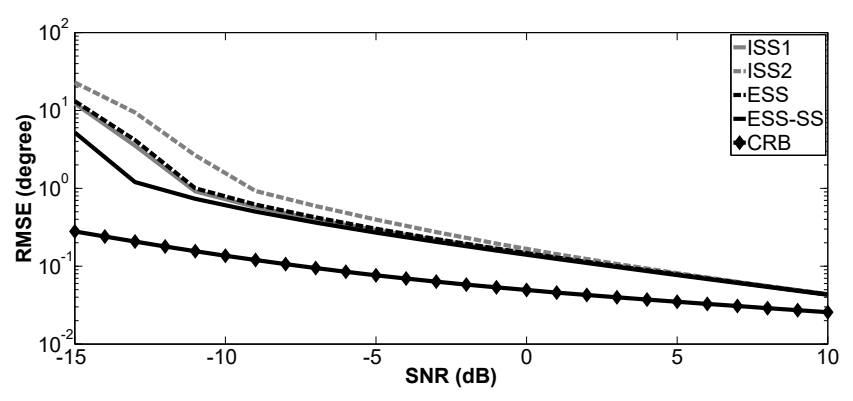

Fig. 3. Case $a$, RMSE of DOA estimation versus SNR.

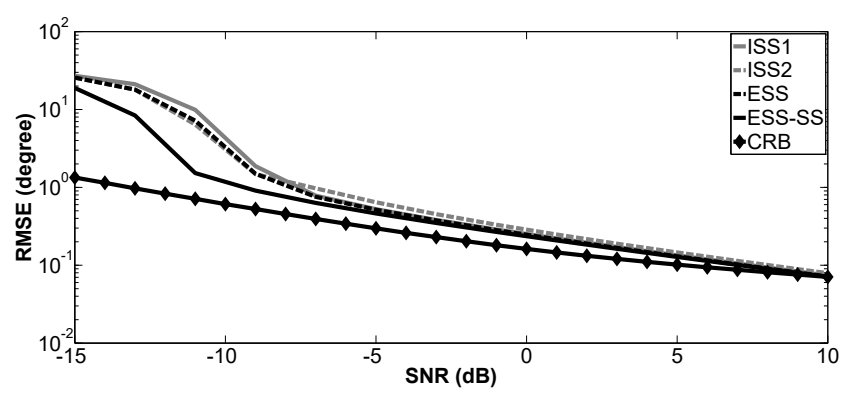

Fig. 4. Case $b$, RMSE of DOA estimation versus SNR.

SNR. For all the methods, it can be seen that the RMSEs continuously decrease when SNR increases. The proposed ESS-SS has a more significant decrease of the RMSE, especially with low SNR. Furthermore, the RMSE of the proposed ESS-SS is smaller (with better accuracy) than that of the other methods at every SNR, which is very close to the CRB with small angle 
separation (Case b), see Fig. 4. In Case $a$ (with large angle separation), the proposed ESS shares a similar performance as ISS1, while ISS2 is inferior to the others. When the angle separation is small, as in Case $b$, the performance of the proposed ESS is slightly better than that of ISS2 and ISS1.

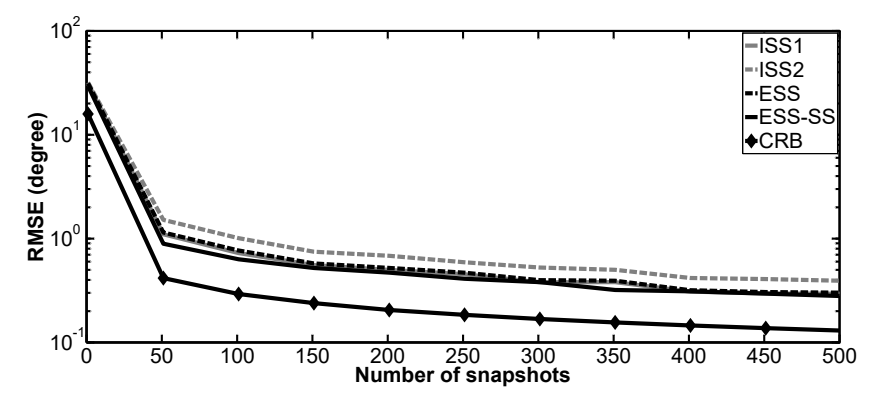

Fig. 5. Case $a$, RMSE of DOA estimation as function of number of snapshots.

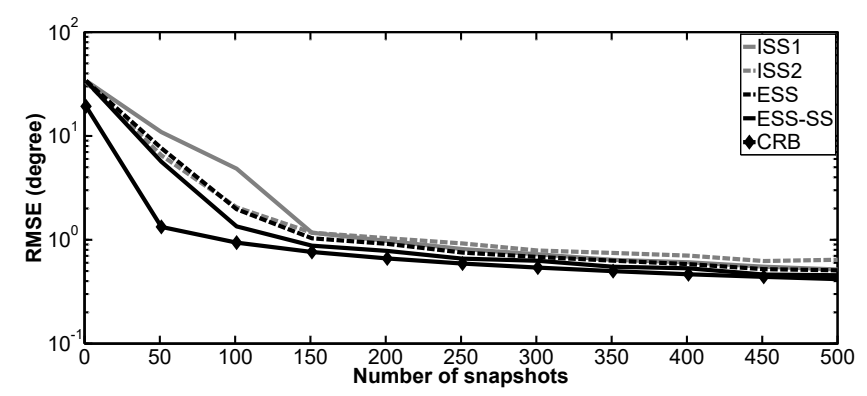

Fig. 6. Case $b$, RMSE of DOA estimation as function of number of snapshots.

Moreover, the performance of the proposed ESS and ESSSS versus the number of snapshots is evaluated. Both Cases $a$ and $b$ are studied with SNR $=-5$ dB. Figs. 5 and 6 plot the RMSEs of the proposed ESS-SS, ESS, ISS1, and ISS2 as function of number of snapshots. According to Figs. 5 and 6, when the number of snapshots is small, the above-mentioned methods cannot work. In addition, with an increasing number of snapshots, the RMSEs decrease. Similar to the results obtained in the second simulation, the proposed ESS-SS offers better performance than that of ESS, ISS1 and ISS2 in both Cases $a$ and $b$. However, ISS1 and ISS2 perform differently when the angle separation changes. When the angle separation is small (Case $b$ ), the performance of ISS1 is worse than that of ISS2; the opposite results can be found with large angle separation (Case $a$ ).

To better understand the influence of angle separation on the performance of the proposed method, in the fourth simulation, the statistical performance of the proposed method versus angle separation between two incoming signals is studied. The relative RMSE (RRMSE) of the estimated DOAs versus angle separation are calculated. The number of Monte-Carlo trials is 400 with 500 independent snapshots. One of the incoming signals is fixed at $\theta_{1}=0^{\circ}$, while the other comes from $\theta_{2}=\theta_{1}+\Delta \theta . \Delta \theta$ varies from $2^{\circ}$ to $15^{\circ}, \mathrm{SNR}=-10 \mathrm{~dB}$. It can be seen from Fig. 7 that all the methods fail to resolve closely spaced incident waves satisfactorily at the beginning. The RRMSEs decrease when the angle separation increases.
The proposed ESS-SS offers the best performance with the smallest RRMSE, particularly when the angle separation is small. The proposed ESS and ISS2 perform similarly (the performance of the proposed ESS is slightly better than that of ISS2). However, the performance of ISS2 degrades rapidly as the angle separation becomes larger. On the contrary, ISS1 provides more biased estimations with small angle separation. As the angle separation becomes greater, ISS1 tends toward similar performance as the proposed ESS and ESS-SS as the curves of RRMSE coincide with each other. In this situation, ISS1 performs better than that of the ISS2.

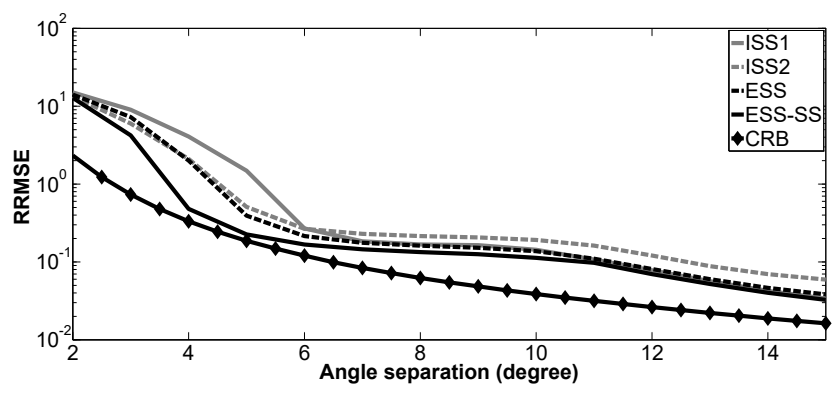

Fig. 7. RRMSE of DOA estimation as function of angle separation.

In the last simulation, the performance of the proposed ESS-SS versus the number of subarrays $M$ is tested with 500 independent runs. The angles in both Cases $a$ and $b$ are applied, SNR is fixed at $10 \mathrm{~dB}$, and $M \in[1,9]$ is due to $N=L+M-1, L>2$. According to Appendix D, the optimal value of $M$ varies with the changing of the DOAs of the incoming signals. It can be seen from Fig. 8, for Case $a$, the RMSE reaches its minimum when $M=7$; for Case $b$, this value $(M)$ is 2 . The simulation results confirm the theoretical analysis in Appendix D. However, the RMSEs are similar with different $M$. In this paper, the number of subarrays $(M)$ is set to 6 .

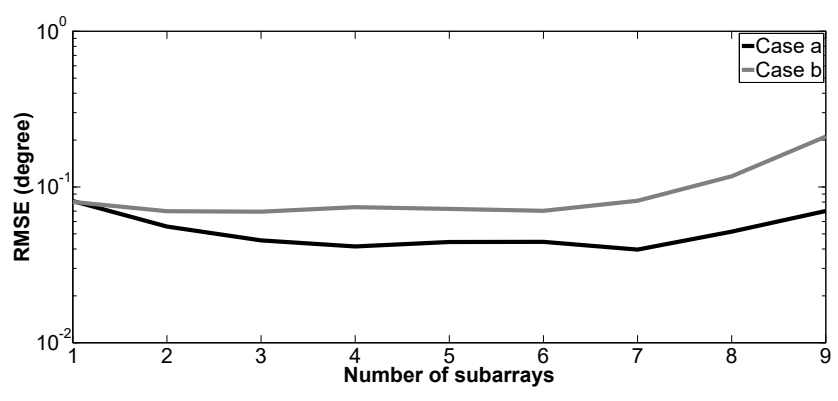

Fig. 8. RMSE of DOA estimation as function of number of subarrays.

\section{Conclusions}

In this paper, an enhanced spatial smoothing technique combined with ESPRIT algorithm is proposed for DOA estimation in coherent scenarios. This new technique takes full advantage of both autocorrelations and cross-correlations of the received signals and can be applied on the signal subspace instead of the entire data covariance matrix. Compared with ISS1 
[25] and ISS2 [26], the proposed ESS and ESS-SS have been proved to be more powerful to de-correlate the correlation between signals and more robust to noise. The performance of the proposed method is tested on numerical data. Simulation results prove that the proposed method improves the stability and robustness of DOA estimation, especially in the scenarios of low SNR, insignificant number of snapshots as well as small angle separation. In the future, we would like to investigate the performance of the proposed method in more practical environment, for example, in the presence of nonuniform noise like in [32], [33], or with real measured data.

\section{ACKNOWLEDGEMENT}

The authors would like to thank the Shanghai Sailing Program (No.19YF1419100) for funding part of this work.

\section{APPENDiX A}

In Appendix $A$, we will prove that the proposed method can restore the rank of the data covariance matrix (the rank of the source covariance matrix after the proposed ESS technique is equal to $K$ ). To facilitate the expression of the proposed ESS technique, the forward only smoothed noiseless covariance matrix $\sum_{i=1}^{M} \sum_{j=1}^{M}\left\{\mathbf{R}_{i j} \mathbf{R}_{j i}+\mathbf{R}_{i i} \mathbf{R}_{j j}\right\}$ is taken as an example.

Firstly, the above matrices can be decomposed as

$$
\begin{aligned}
& \sum_{i=1}^{M} \sum_{j=1}^{M}\left\{\mathbf{R}_{i j} \mathbf{R}_{j i}\right\}= \\
& \sum_{i=1}^{M} \sum_{j=1}^{M}\left\{\mathbf{A}_{1} \mathbf{D}^{i-1} \mathbf{S}\left(\mathbf{D}^{j-1}\right)^{H} \mathbf{A}_{1}^{H} \mathbf{A}_{1} \mathbf{D}^{j-1} \mathbf{S}\left(\mathbf{D}^{i-1}\right)^{H} \mathbf{A}_{1}^{H}\right\} \\
& =\sum_{i=1}^{M} \sum_{j=1}^{M} \Psi_{j j}\left\{\mathbf{A}_{1} \mathbf{D}^{i-1} \mathbf{S}\left(\mathbf{D}^{i-1}\right)^{H} \mathbf{A}_{1}^{H}\right\} \\
& =\mathbf{A}_{1} \overline{\mathbf{S}}_{1} \mathbf{A}_{1}^{H} \\
& \sum_{i=1}^{M} \sum_{j=1}^{M}\left\{\mathbf{R}_{i i} \mathbf{R}_{j j}\right\}= \\
& \sum_{i=1}^{M} \sum_{j=1}^{M}\left\{\mathbf{A}_{1} \mathbf{D}^{i-1} \mathbf{S}\left(\mathbf{D}^{i-1}\right)^{H} \mathbf{A}_{1}^{H} \mathbf{A}_{1} \mathbf{D}^{j-1} \mathbf{S}\left(\mathbf{D}^{j-1}\right)^{H} \mathbf{A}_{1}^{H}\right\} \\
& =\sum_{i=1}^{M} \sum_{j=1}^{M} \Psi_{i j}\left\{\mathbf{A}_{1} \mathbf{D}^{i-1} \mathbf{S}\left(\mathbf{D}^{j-1}\right)^{H} \mathbf{A}_{1}^{H}\right\} \\
& =\mathbf{A}_{1} \overline{\mathbf{S}}_{2} \mathbf{A}_{1}^{H}
\end{aligned}
$$

where $\Psi_{i j}=\mathbf{p}^{H}\left(\mathbf{D}^{i-1}\right)^{H} \mathbf{A}_{1}^{H} \mathbf{A}_{1} \mathbf{D}^{j-1} \mathbf{p}$ is real, and $\mathbf{S}=$ $\mathbf{p} \mathbf{p}^{H}$. It has been proved in [26], the source covariance matrix
$\overline{\mathbf{S}}_{1}$ or $\overline{\mathbf{S}}_{2}$ is of rank $K$. In addition,

$$
\begin{aligned}
& \mathbf{A}_{1} \overline{\mathbf{S}}_{i j} \mathbf{A}_{1}^{H} \\
& =\mathbf{A}_{1}\left\{\sum_{i=1}^{M} \sum_{j=1}^{M} \Psi_{i j}\left\{\mathbf{D}^{i-1} \mathbf{S}\left(\mathbf{D}^{j-1}\right)^{H}\right\}\right\} \mathbf{A}_{1}^{H} \\
& =\mathbf{A}_{1}\left\{\sum_{i=1}^{M}\left\{\psi_{i} \mathbf{D}^{i-1} \mathbf{p}\right\} \sum_{j=1}^{M}\left\{\psi_{j}^{*}\left(\mathbf{D}^{j-1} \mathbf{p}\right)^{H}\right\}\right\} \mathbf{A}_{1}^{H} \\
& =\mathbf{A}_{1}\left\{\sum_{i=1}^{M}\left\{\psi_{i} \mathbf{D}^{i-1} \mathbf{p}\right\} \sum_{i=1}^{M}\left\{\psi_{i}^{*}\left(\mathbf{D}^{i-1} \mathbf{p}\right)^{H}\right\}\right\} \mathbf{A}_{1}^{H},
\end{aligned}
$$

where $\Psi_{i j}=\psi_{i} \psi_{j}^{*}$. Therefore, the noiseless matrices $\sum_{i=1}^{M} \sum_{j=1}^{M}\left\{\mathbf{R}_{i j} \mathbf{R}_{j i}\right\}$ and $\sum_{i=1}^{M} \sum_{j=1}^{M}\left\{\mathbf{R}_{i i} \mathbf{R}_{j j}\right\}$ are positive semidefinite matrices. Due to

$$
\begin{aligned}
& \sum_{i=1}^{M} \sum_{j=1}^{M}\left\{\mathbf{R}_{i j} \mathbf{R}_{j i}+\mathbf{R}_{i i} \mathbf{R}_{j j}\right\} \\
& =\mathbf{A}_{1}\left\{\overline{\mathbf{S}}_{1}+\overline{\mathbf{S}}_{2}\right\} \mathbf{A}_{1}^{H}
\end{aligned}
$$

we can conclude that the source covariance matrix $\left\{\overline{\mathbf{S}}_{1}+\overline{\mathbf{S}}_{2}\right\}$ is also of rank $K$ and the noiseless matrix $\sum_{i=1}^{M} \sum_{j=1}^{M}\left\{\mathbf{R}_{i j} \mathbf{R}_{j i}+\mathbf{R}_{i i} \mathbf{R}_{j j}\right\}$ is positive semidefinite. In addition, the above proof can easily be extended to the forward-backward smoothed covariance matrix.

\section{APPENDIX B}

In this appendix, we will prove that the rank of $\mathbf{R}_{1}^{f}+\mathbf{R}_{2}^{f}$ equals to $K$. To begin with, define $\Phi_{i j}=$ $\mathbf{t}^{H}\left(\mathbf{D}^{i-1}\right)^{H} \mathbf{A}_{1}^{H} \mathbf{A}_{1} \mathbf{D}^{j-1} \mathbf{t}$. We can deduce that

$$
\begin{aligned}
& \mathbf{R}_{1}^{f}+\mathbf{R}_{2}^{f}= \\
& \mathbf{A}_{1}\left\{\sum_{i=1}^{M} \sum_{j=1}^{M} \Phi_{i j}\left\{\mathbf{D}^{i-1} \mathbf{t t}^{H}\left(\mathbf{D}^{j-1}\right)^{H}\right\}\right. \\
& \left.+\Phi_{j j}\left\{\mathbf{D}^{i-1} \mathbf{t t}^{H}\left(\mathbf{D}^{i-1}\right)^{H}\right\}\right\} \mathbf{A}_{1}^{H} .
\end{aligned}
$$

Therefore, we need prove $\left\{\sum_{i=1}^{M} \sum_{j=1}^{M} \Phi_{i j} \mathbf{D}^{i-1} \mathbf{t t}^{H}\left(\mathbf{D}^{j-1}\right)^{H}\right.$ $\left.+\Phi_{j j} \mathbf{D}^{i-1} \mathbf{t t}^{H}\left(\mathbf{D}^{i-1}\right)^{H}\right\}$ is not singular, which is exactly the same as the case in Appendix $A$. The only difference is that we use $\mathbf{t t}^{H}$ instead of $\mathbf{S}$ as in Appendix $A$. Therefore, to finish our proof, we must prove that the Hermitian square root $\mathbf{C}(\mathbf{C}$ is a $(K \times K)$-dimensional matrix with $\left.\mathbf{C} \mathbf{C}^{H}=\mathbf{t t}^{H}\right)$ of $\mathbf{t t}^{H}$ should have at least one nonzero element in each row. For coherent signals, the signal eigenvector $\mathbf{u}$ is a linear combination of the directional vectors $\mathbf{a}\left(\theta_{1}\right), \mathbf{a}\left(\theta_{2}\right), \ldots, \mathbf{a}\left(\theta_{K}\right)$ with $\mathbf{u}=$ $k_{1} \mathbf{a}\left(\theta_{1}\right)+k_{2} \mathbf{a}\left(\theta_{2}\right)+\ldots+k_{K} \mathbf{a}\left(\theta_{K}\right)=\mathbf{A t}, k_{1}, k_{2}, \ldots, k_{K} \neq 0$, and $\mathbf{t}=\left[\begin{array}{llll}k_{1} & k_{2} & \cdots & k_{K}\end{array}\right]^{T}$ [1]. The elements inside $\mathbf{t t}^{H}$ are non-zero, and we can prove that the Hermitian square root $\mathbf{C}$ of $\mathbf{t t}^{H}$ has at least one nonzero element in each row. Therefore, the rank of $\left\{\sum_{i=1}^{M} \sum_{j=1}^{M} \Phi_{i j} \mathbf{D}^{i-1} \mathbf{t t}^{H}\left(\mathbf{D}^{j-1}\right)^{H}\right.$ $\left.+\Phi_{j j} \mathbf{D}^{i-1} \mathbf{t t}^{H}\left(\mathbf{D}^{i-1}\right)^{H}\right\}$ is $K$.

To conclude, the forward smoothed covariance matrix $\mathbf{R}_{\text {ESS-SS }}^{f}$ constructed by the proposed ESS-SS technique has the same rank as the noise free data covariance matrix for the totally uncorrelated or partly correlated cases. 


\section{APPENDIX C}

In this appendix, the expression of matrix $\mathbf{X}$ will be given. After applying the proposed de-correlation procedure, the new data covariance matrix $\mathbf{R}_{\text {ESS-ss }}$ can be calculated. Define the signal eigenvectors of $\mathbf{R}_{\mathrm{ESS}-\mathrm{sS}}$ are in the columns of $\mathbf{V}$. As mentioned in [1], the matrices $\mathbf{A}_{1}$ and $\mathbf{V}$ span the same range space when the source covariance matrix is full rank. Therefore, there exists a full rank matrix $\mathbf{T}$ with $(K \times K)$ dimensions:

$$
\mathbf{V}=\mathbf{A}_{1} \mathbf{T}
$$

Define

$$
\mathbf{V}=\left(\begin{array}{c}
\mathbf{V}_{1} \\
\text { last row }
\end{array}\right)=\left(\begin{array}{c}
\text { first row } \\
\mathbf{V}_{2}
\end{array}\right)
$$

it can be deduced that

$$
\begin{aligned}
& \mathbf{A} \uparrow \mathbf{T}=\mathbf{V}_{1} \\
& \mathbf{A} \downarrow \mathbf{T}=\mathbf{A} \uparrow \mathbf{D T}=\mathbf{V}_{2},
\end{aligned}
$$

we can get

$$
\mathbf{V}_{1} \mathbf{T}^{-1} \mathbf{D} \mathbf{T}=\mathbf{V}_{1} \mathbf{X}=\mathbf{V}_{2}
$$

Therefore,

$$
\mathbf{X}=\mathbf{V}_{1}^{+} \mathbf{V}_{2},
$$

where + is the Moore-Penrose inverse operator. Note that $\mathbf{X}$ and $\mathbf{D}$ are related by a similarity transformation, therefore they have the same eigenvalues.

\section{APPENDIX D}

In this appendix, we will give the theoretical analysis of the optimal $M$. Only the forward only smoothed covariance matrix $\mathbf{R}_{\mathrm{ESS}-\mathrm{SS}}^{f}$ is studied. According to the results in Appendix B, $\mathbf{R}_{\text {ESS-SS }}^{f}$ can be expressed as

$$
\begin{aligned}
& \mathbf{R}_{1}^{f}+\mathbf{R}_{2}^{f}= \\
& \mathbf{A}_{1}\left\{\sum_{i=1}^{M} \sum_{j=1}^{M} \Phi_{i j} \mathbf{D}^{i-1} \mathbf{t t}^{H}\left(\mathbf{D}^{j-1}\right)^{H}\right. \\
& \left.+\Phi_{j j} \mathbf{D}^{i-1} \mathbf{t t}^{H}\left(\mathbf{D}^{i-1}\right)^{H}\right\} \mathbf{A}_{1}^{H} .
\end{aligned}
$$

where $\Phi_{i j}$ and $\Phi_{j j}$ are scalar. Define $\mathbf{S}_{\mathrm{ESS}-\mathrm{SS}}=\left\{\sum_{i=1}^{M} \sum_{j=1}^{M} \Phi_{i j} \mathbf{D}^{i-1} \mathbf{t t}^{H}\left(\mathbf{D}^{j-1}\right)^{H}+\right.$ $\left.\Phi_{j j} \mathbf{D}^{i-1} \mathbf{t t}^{H}\left(\mathbf{D}^{i-1}\right)^{H}\right\}$, matrix $\mathbf{R}_{\mathrm{ESS}-\text { SS }}^{f}$ can be rewritten as

$$
\mathbf{R}_{\mathrm{ESS}-\mathrm{SS}}^{f}=\mathbf{A}_{1} \mathbf{S}_{\mathrm{ESS}-\mathrm{SS}} \mathbf{A}_{1}^{H} \text {. }
$$

Define $\mathbf{S}_{\mathrm{ESS}-\mathrm{SS}}(m, n)$ as the $(m, n)$ th element of $\mathbf{S}_{\mathrm{ESS}-\mathrm{sS}}$, $\mathbf{t}(m)$ and $\mathbf{t}(n)$ are the $m$ th and $n$th elements of vector t, respectively. According to [19], $\frac{\mathbf{S}_{\mathrm{ESS}-\mathrm{sS}}(m, n)}{\mathbf{t}(m) \mathbf{t}^{*}(n)}$ represents the effective correlation coefficient between the $m$ th and $n$th received signals after decorrelation, which can be expressed as

$$
\begin{aligned}
& \frac{\mathbf{S}_{\mathrm{ESS}-\mathrm{SS}}(m, n)}{\mathbf{t}(m) \mathbf{t}^{*}(n)} \\
& =\sum_{i=1}^{M} \sum_{j=1}^{M}\left\{\Phi_{i j} \exp \left\{-j 2 \pi \frac{d}{\lambda} \sin \theta_{m}\right\}^{i} \exp \left\{j 2 \pi \frac{d}{\lambda} \sin \theta_{n}\right\}^{j}\right. \\
& \left.+\Phi_{j j} \exp \left\{j 2 \pi \frac{d}{\lambda}\left(\sin \theta_{n}-\sin \theta_{m}\right)\right\}^{i}\right\} .
\end{aligned}
$$

From the above equation, it is clear that the decorrelation effect of the proposed method depends on the number of subarrays $(M)$ and DOAs of signals. Given $\theta_{m}$ and $\theta_{n}$ of the $m$ th and $n$th received signals, the optimal value of $M\left(\widehat{M}_{o p t}\right)$ can be deduced from the following equation:

$$
\widehat{M}_{\text {opt }}=\underset{M}{\arg \min }\left|\frac{\mathbf{S}_{\mathrm{ESS}-\mathrm{SS}}(m, n)}{\mathbf{t}(m) \mathbf{t}^{*}(n)}\right| .
$$

The above complex optimization problem can be solved by the exhaustive search of the minimal value of $\left|\frac{\mathbf{S}_{\mathrm{ESS}-\mathrm{SS}}(m, n)}{\mathbf{t}(m) \mathbf{t}^{*}(n)}\right|$.

\section{REFERENCES}

[1] H. Krim and M. Viberg, "Two decades of array signal processing research: the parametric approach," IEEE signal processing magazine, vol. 13, no. 4, pp. 67-94, 1996.

[2] T. E. Tuncer and B. Friedlander, Classical and modern direction-ofarrival estimation. Academic Press, 2009.

[3] M. Sun, J. Pan, C. Le Bastard, Y. Wang, and J. Li, "Advanced signal processing methods for ground-penetrating radar: Applications to civil engineering," IEEE Signal Processing Magazine, vol. 36, no. 4, pp. 7484, 2019.

[4] M. Jin, G. Liao, and J. Li, "Joint DOD and DOA estimation for bistatic MIMO radar," Signal Processing, vol. 89, no. 2, pp. 244-251, 2009.

[5] S. Geng, J. Kivinen, X. Zhao, and P. Vainikainen, "Millimeter-wave propagation channel characterization for short-range wireless communications," IEEE Transactions on Vehicular Technology, vol. 58, no. 1, pp. 3-13, 2009.

[6] R. O. Schmidt, "Multiple emitter location and signal parameter estimation," IEEE Transactions on Antennas and Propagation, vol. 34, no. 3, pp. 276-280, 1986.

[7] R. Roy and T. Kailath, "ESPRIT-estimation of signal parameters via rotational invariance techniques," IEEE Transactions on Acoustics, Speech and Signal Processing, vol. 37, no. 7, pp. 984-995, 1989.

[8] Y. Hua and T. K. Sarkar, "Matrix pencil method for estimating parameters of exponentially damped/undamped sinusoids in noise," IEEE Transactions on Acoustics, Speech, and Signal Processing, vol. 38, no. 5, pp. 814-824, 1990.

[9] M. Viberg and B. Ottersten, "Sensor array processing based on subspace fitting," IEEE Transactions on signal processing, vol. 39, no. 5, pp. 1110-1121, 1991.

[10] M. Viberg, B. Ottersten, and T. Kailath, "Detection and estimation in sensor arrays using weighted subspace fitting," IEEE transactions on Signal Processing, vol. 39, no. 11, pp. 2436-2449, 1991.

[11] C. J, "High-resolution frequency-wavenumber spectrum analysis," Proceedings of the IEEE, vol. 57, no. 8, pp. 1408-1418, 1969.

[12] J. Pan, Y. Wang, C. L. Bastard, and T. Wang, "DOA finding with support vector regression based forwardbackward linear prediction," Sensors, vol. 17, no. 6, p. 1225, 2017.

[13] S. Marcos, A. Marsal, and M. Benidir, "The propagator method for source bearing estimation," Signal processing, vol. 42, no. 2, pp. 121138, 1995

[14] T. J. Shan, M. Wax, and T. Kailath, "On spatial smoothing for direction of arrival estimation of coherent signals," IEEE Transactions on Acoustics, Speech and Signal Processing, vol. 33, no. 4, pp. 806-811, 1985.

[15] I. Ziskind and M. Wax, "Maximum likelihood localization of multiple sources by alternating projection," IEEE Trans.acoust.speech and Signal Processing, vol. 36, no. 10, pp. 1553-1560. 
[16] M. Zoltowski and F. Haber, "A vector space approach to direction finding in a coherent multipath environment," IEEE Transactions on Antennas and Propagation, vol. 34, no. 9, pp. 1069-1079, 1986.

[17] J. E. Evans, D. F. Sun, and J. R. Johnson, "Application of advanced signal processing techniques to angle of arrival estimation in ATC navigation and surveillance systems," Calculation, 1982.

[18] S. U. Pillai and B. H. Kwon, "Forward/backward spatial smoothing techniques for coherent signal identification," IEEE Transactions on Acoustics, Speech and Signal Processing, vol. 37, no. 1, pp. 8-15, 1989.

[19] H. Yamada, M. Ohmiya, Y. Ogawa, and K. Itoh, "Superresolution techniques for time-domain measurements with a network analyzer," IEEE Transactions on Antennas and Propagation, vol. 39, no. 2, pp. 177-183, 1991.

[20] R. T. Williams, S. Prasad, A. K. Mahalanabis, and L. H. Sibul, "An improved spatial smoothing technique for bearing estimation in a multipath environment," IEEE Transactions on Acoustics, Speech and Signal Processing, vol. 36, no. 4, pp. 425-432, 1988.

[21] G. T. Pham, P. Loubaton, and P. Vallet, "Performance analysis of spatial smoothing schemes in the context of large arrays," IEEE transactions on Signal Processing, vol. 64, no. 1, pp. 160-172, 2016.

[22] F. M. Han and X. D. Zhang, "An ESPRIT-like algorithm for coherent DOA estimation," IEEE Antennas and Wireless Propagation Letters, vol. 4, no. 1, pp. 443-446, 2005.

[23] Z. Ye and X. Xu, "DOA estimation by exploiting the symmetric configuration of uniform linear array," IEEE Transactions on Antennas and Propagation, vol. 55, no. 12, pp. 3716-3720, 2007.

[24] X. Zhang, J. Yu, G. Feng, and D. Xu, "Blind direction of arrival estimation of coherent sources using multi-invariance property," Progress In Electromagnetics Research, vol. 88, pp. 181-195, 2008.

[25] W. Du and R. L. Kirlin, "Improved spatial smoothing techniques for DOA estimation of coherent signals," IEEE Transactions on Signal Processing, vol. 39, no. 5, pp. 1208-1210, 1991.

[26] M. Dong, S. Zhang, X. Wu, and Z. Huanying, "A high resolution spatial smoothing algorithm," in IEEE International Symposium on Microwave, Antenna, Propagation, and EMC Technologies For Wireless Communications, pp. 1031-1034, 2007.

[27] L. Qu, Q. Sun, T. Yang, L. Zhang, and Y. Sun, "Time-delay estimation for ground penetrating radar using ESPRIT with improved spatial smoothing technique," IEEE Geoscience and Remote Sensing Letters, vol. 11, no. 8, pp. 1315-1319, 2014.

[28] M. Sun, C. L. Bastard, Y. Wang, and N. Pinel, "Time-delay estimation using ESPRIT with extended improved spatial smoothing techniques for radar signals," IEEE Geoscience and Remote Sensing Letters, vol. 13, no. 1, pp. 73-77, 2016.

[29] P. Protiva, J. Mrkvica, and J. Machác, "Estimation of wall parameters from time-delay-only through-wall radar measurements," IEEE Transactions on Antennas and Propagation, vol. 59, no. 11, pp. 4268-4278, 2011.

[30] P. Protiva, J. Mrkvica, and J. Macháč, "Time delay estimation of UWB radar signals backscattered from a wall," Microwave and optical technology letters, vol. 53, no. 6, pp. 1444-1450, 2011.

[31] P. Stoica, G. Larsson, and A. B. Gershman, "The stochastic CRB for array processing: a textbook derivation," IEEE Signal Processing Letters, vol. 8, no. 5, pp. 148-150, 2008.

[32] C. E. Chen, F. Lorenzelli, R. E. Hudson, and K. Yao, "Stochastic maximum-likelihood DOA estimation in the presence of unknown nonuniform noise," IEEE Transactions on Signal Processing, vol. 56, no. 7, pp. 3038-3044, 2008.

[33] A. K. Seghouane, "A Kullback-Leibler methodology for unconditional ML DOA estimation in unknown nonuniform noise," IEEE Transactions on Aerospace and Electronic Systems, vol. 47, no. 4, pp. 3012-3021, 2011. 